\title{
Repacking on demand for speed-sensitive channel assignment
}

\author{
Hsien-Ming Tsai ${ }^{a}$, Ai-Chun Pang ${ }^{b}$, Yung-Chun Lin ${ }^{c}$, Yi-Bing Lin ${ }^{c, d, *}$ \\ ${ }^{a}$ Quanta Research Institute, Quanta Computer Inc., No. 4, Wen Ming 1 St., Kuei Shan Hsiang, Tao Yuan Shien, Taiwan \\ ${ }^{\mathrm{b}}$ Graduate Institute of Networking and Multimedia, Department of Computer Science and Information Engineering, \\ National Taiwan University, No. 1, Sec. 4, Roosevelt Rd., Taipei, Taiwan \\ ${ }^{\mathrm{c}}$ Department of Computer Science and Information Engineering, National Chiao Tung University, 1001 Ta Hsueh Rd., Hsinchu, Taiwan \\ d Providence University, Chung-chi Rd., Shalu Taichung, Taiwan
}

Received 17 December 2003; received in revised form 27 July 2004; accepted 27 July 2004

Available online 14 October 2004

Responsible Editor: E. Chong

\begin{abstract}
In mobile telecommunications networks, radio channels are limited resources that should be carefully allocated. To increase network capacity, channel assignment can be exercised in a hierarchical cellular network (HCN). By considering the speeds of MSs, we propose an HCN channel assignment approach called repacking on demand (RoD). A simulation model is developed to investigate the performance of RoD and some previously proposed approaches for HCN. Our study quantitatively shows that $\mathrm{RoD}$ significantly outperforms the previous proposed approaches.

(C) 2004 Elsevier B.V. All rights reserved.
\end{abstract}

Keywords: Channel assignment; Channel repacking; Hierarchical cellular network; Repacking on demand

\section{Introduction}

One of the most important issues in cellular network operation is capacity planning. Especially when subscriber population grows rapidly, a cellular service provider needs to increase its network capacity effectively. One possible solution is to deploy

\footnotetext{
* Corresponding author. Address: Department of Computer Science and Information Engineering, National Chiao Tung University, 1001 Ta Hsueh Rd., Hsinchu, Taiwan. Tel.: +886 3 5731842; fax: +886 35724176 .

E-mail address: liny@csie.nctu.edu.tw (Y.-B. Lin).
}

hierarchical cellular network (HCN) $[6,11,13]$. As shown in Fig. 1, an HCN consists of two types of base stations (BSs): micro BSs and macro BSs. A micro BS with low power transceivers provides small radio coverage (referred to as microcell), and a macro BS with high power transceivers provides large radio coverage (referred to as macrocell). The microcells cover mobile stations (MSs) in heavy teletraffic areas. A macrocell is overlaid with several microcells to cover all MSs in these microcells.

In an HCN, radio channels must be carefully assigned to reduce the numbers of new call blockings 

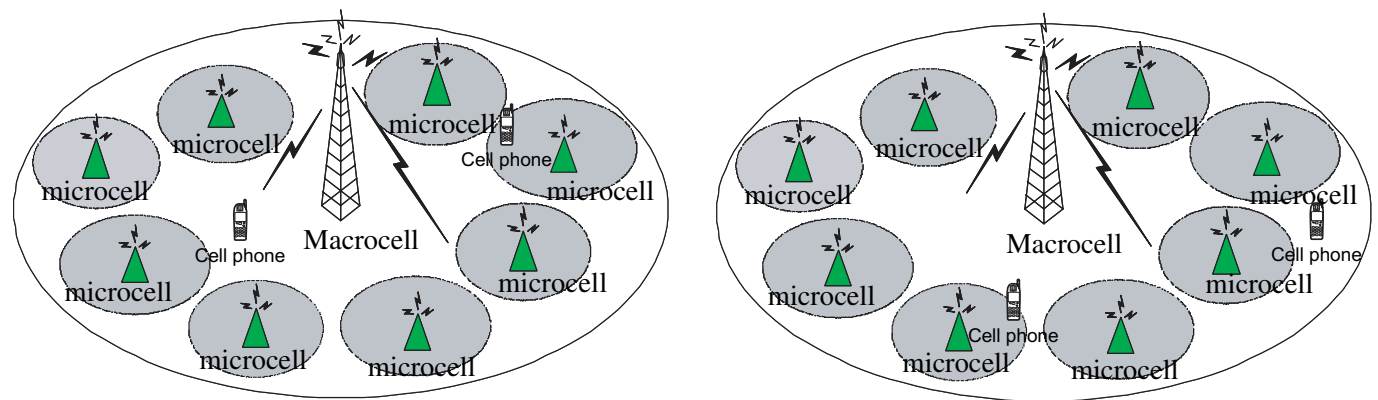

Fig. 1. Hierarchical cellular network architecture with two macrocells.

as well as handoff call force-terminations. Several channel planning and assignment approaches have been proposed for HCN [1,5,18,14-17]. Some of them assign channels according to the received radio signal strength $[5,15]$. Other approaches $[1,14,16,17]$ reduce call blocking and force-termination through repacking techniques. Repacking is the process of switching a connected call from a macrocell to a microcell and vice versa. Based on the repacking techniques, our previous work proposed Repacking on Demand (RoD) [7,18] to improve the network performance. RoD was originally designed to efficiently allocate the radio channels for wireless local loop (WLL) systems [7]. In [18], we studied the RoD performance for mobile networks without considering the MS speeds. In this paper, we investigate repacking performance improvement by considering the moving speeds of MSs.

Speed-sensitive channel assignment and repacking (including Macro-to-micro and micro-to-Macro repacking) are exercised to satisfy the following criteria:

Criterion 1. Calls for slow MSs tend to be assigned with microcell channels so that the "global resources" of macrocells can be effectively shared by calls in the blocked microcells (i.e., the microcells without any idle channel).

Criterion 2. Calls for fast MSs tend to be assigned with macrocell channels so that the number of handoffs can be reduced.

Following Criterion 1, Macro-to-micro (Mto-m) repacking may be exercised to switch a call for a slow MS from the macrocell to the microcell.
In this case, the "global resources" of macrocells can be effectively shared by calls in the blocked microcells. Following Criterion 2, the microto-Macro (m-to-M) repacking may be exercised to switch a call for a fast MS from the microcell to the macrocell. In this case, the number of handoffs can be reduced.

In this paper, we consider several speed-sensitive channel assignment and repacking approaches for HCN: Always Repacking (AR; or take-back in $[1,8,14])$ always exercises repacking as soon as some specific events (e.g., a channel is released at the micro/macrocell) occur to reduce the number of handoffs for calls of fast MSs. However, always exercising repacking may degrade the system performance on call blocking probability and force-termination probability in some cases (e.g., the number of macrocell channels is small). In Partial Repacking on Demand (PRoD; or preemption in $[19,20])$, only calls with slow speeds are repacked on demand. When the proportion of fast calls increases, the effect of slow-call repacking on performance improvement becomes insignificant. Therefore, in order to further reduce call blocking and force-termination, this paper improves the Repakcing on Demand (RoD) approach by considering repacking for both slow and fast MSs.

This paper is organized as follows. In Section 2, we describe speed-sensitive HCN channel assignment approaches including AR, PRoD and RoD. In Section 3, input parameters and output measures for these channel assignment approaches are described. Section 4 compares RoD with AR and PRoD. Our study quantitatively shows that RoD outperforms AR and PRoD. 


\section{Speed-sensitive channel assignment for $\mathrm{HCN}$}

Based on the moving speeds of MSs, several speed-sensitive channel assignment approaches have been proposed. In these approaches, a call for a fast MS is referred to as a fast call, and a call for a slow MS is referred to as a slow call.

No Repacking (NR) [16]: This approach does not perform repacking. Based on Criteria 1 and 2, the slow and fast calls are handled differently.

- NR for Slow Calls (Fig. 2). When a slow call attempt is newly generated at or handed off to the $i$ th microcell, the $\mathrm{HCN}$ first tries to assign a channel in the $i$ th microcell (following Criterion 1) to the call attempt (Steps 1 and 2 in Fig. 2). If no idle channel is available in the $i$ th microcell, the call attempt overflows to the macrocell that is overlaid with the $i$ th microcell. If the macrocell has an idle channel, the HCN accepts the call (Steps 3 and 4). Otherwise, the call attempt is rejected; i.e., the new call is blocked or the handoff call is forced to terminate (Step 5). Steps 1-4 in Fig. 2 are called the NR Slow MS Channel Assignment Procedure.

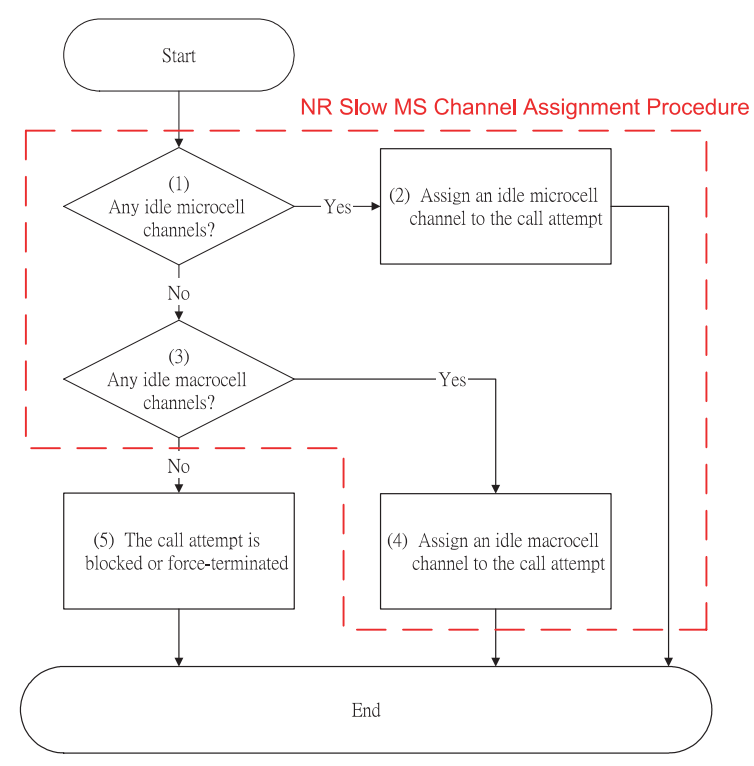

Fig. 2. No repacking for slow MSs.
- NR for Fast Calls (Fig. 3). When a new or handoff fast call attempt occurs in the ith microcell, the HCN first tries to assign the call attempt a channel in the macrocell (following Criterion 2) that is overlaid with the $i$ th microcell (Steps 1 and 2 in Fig. 3). If no idle channel is available in the macrocell, the call attempt overflows to the $i$ th microcell, and the HCN tries to allocate the call a channel in the $i$ th microcell (Steps 3 and 4). If no idle channel is available in the microcell, the call attempt is rejected (Step 5). When a call is complete or the MS moves out of a cell, the radio channel is reclaimed to the idle channel pool of the corresponding micro (macro) cell.

Always Repacking (AR) $[1,8,14]$ : The repacking mechanism is triggered when an occupied channel is released. Two repacking procedures are exercised in AR to reduce the force-termination probability of fast calls. In Macro-to-micro (M-to-m) repacking, a slow call occupying a macrocell channel is switched to an idle channel in the microcell where the MS resides (following Criterion 1). In micro-to-Macro (m-to-M) repacking, a fast call occupying a microcell channel is switched to an

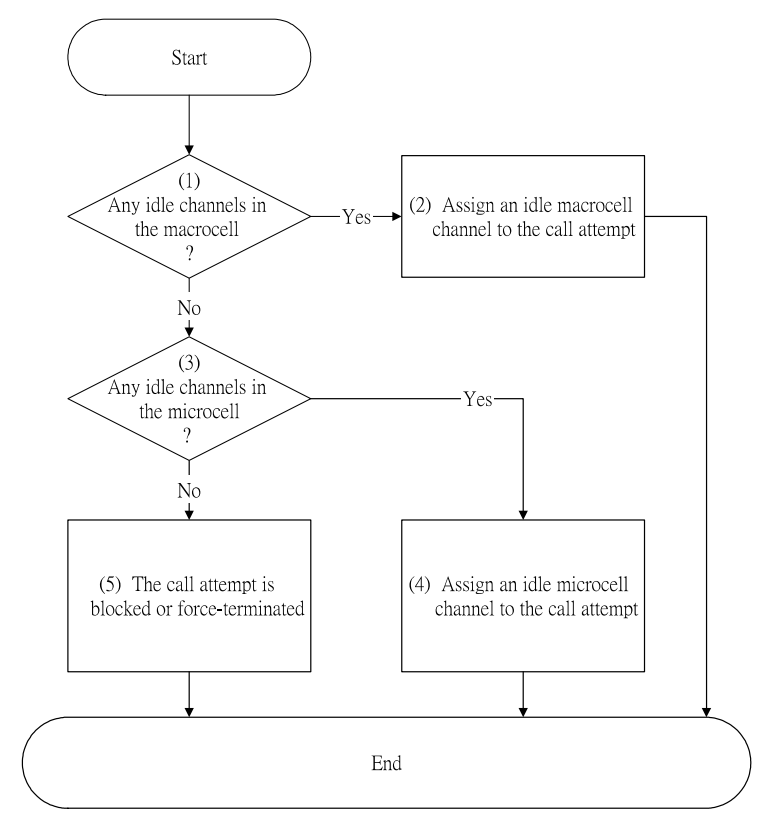

Fig. 3. No repacking for fast MSs. 
idle channel of the overlay macrocell (following Criterion 2). During repacking, one or more calls may be available for switching. These calls are referred to as the repacking candidates. The AR channel assignment is the same as that for NR (see Figs. 2 and 3). The AR repacking procedures (Fig. 4(a) and (b)) are repeatedly executed to check if one of the following situations occurs.

M-to-m Repacking (Fig. 4(a)): When a channel is released at a microcell (Step 1 in Fig. 4(a)), the HCN checks if there is any slow M-to-m repacking candidate (which is a slow call) in

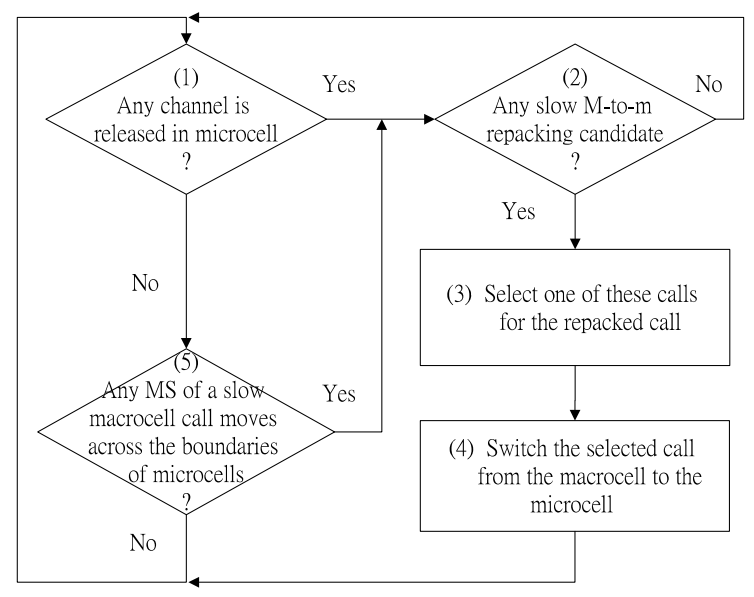

(a)

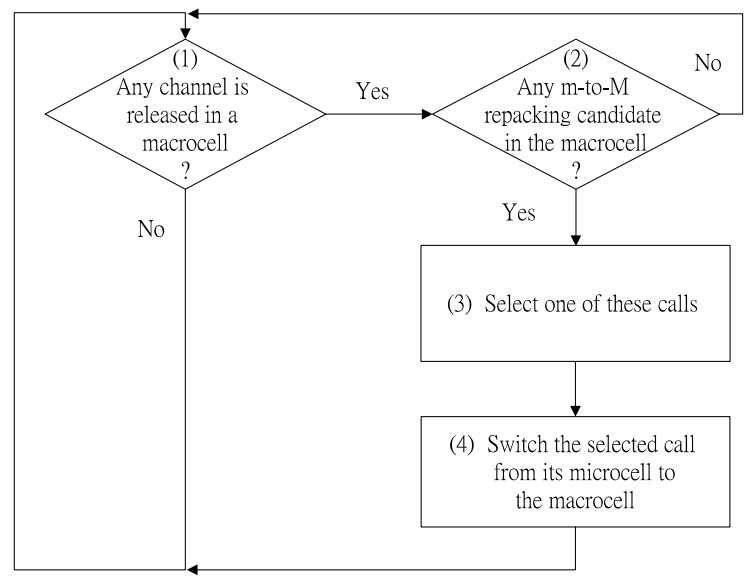

(b)

Fig. 4. Channel repacking procedures for AR (a) M-to-m repacking procedure for $\mathrm{AR}$ (b) $\mathrm{m}$-to-M repacking procedure for AR. the corresponding macrocell (Step 2). If so, M-to-m repacking is exercised to switch one of the slow repacking candidates from the macrocell to the microcell (Steps 3 and 4).

When an MS of a slow macrocell call moves across the boundary of two microcells in that macrocell (Step 5 in Fig. 4(a)), the HCN checks if the call can be a slow M-to-m repacking candidate (Step 2 in Fig. 4(a)). If so, Steps 3 and 4 in Fig. 4(a) are executed to perform M-to-m repacking.

m-to-M Repacking (Fig. 4(b)): When a channel is released in a macrocell (Step 1 in Fig. 4(b)), the $\mathrm{HCN}$ checks if there is any m-to-M repacking candidate (which is a fast call ; Step 2). If so, $\mathrm{m}$-to-M repacking is exercised (Steps 3 and 4).

The following approach is proposed in this paper.

Repacking on Demand ( RoD): In RoD, the HCN may trigger the M-to-m repacking when a call assignment (for a new call or a handoff call) occurs (following Criterion 1). Note that Criterion 2 is used in channel assignment for fast calls, but is not used in repacking.

- RoD I: RoD for Slow Calls (Fig. 5).

Step RoD I.1: When a slow call attempt $C_{\mathrm{s}}$ is newly generated at or handed off to the $i$ th microcell, the HCN first exercises the NR slow MS channel assignment procedure. If the call attempt $C_{\mathrm{s}}$ is assigned a channel, the procedure exits.

Step RoD I.2: If no idle channel is found in Step RoD I.1, then following Criterion 1, the $\mathrm{HCN}$ checks if there is any slow M-to-m repacking candidate. If so, Step RoD I.3 is executed. Otherwise, Step RoD I.5 is executed.

Steps RoD I.3 and 4: The HCN selects one of these calls to exercise M-to-m repacking, and the reclaimed macrocell channel is assigned to the slow call attempt $C_{\mathrm{s}}$. The procedure exits. Steps RoD I.5 and 6: If no slow M-to-m repacking candidate is found, the HCN checks if there is any fast M-to-m repacking candidate. If so, go to Step RoD I.3. Otherwise, the call attempt $C_{\mathrm{s}}$ is rejected. 


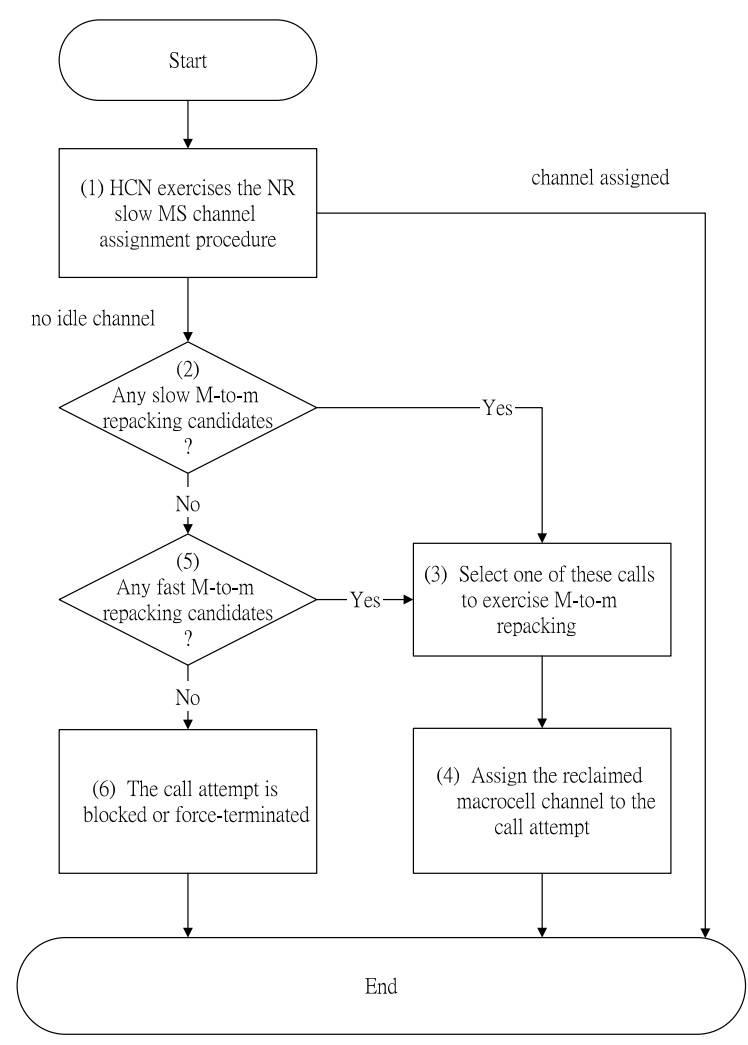

Fig. 5. RoD I: RoD for slow MSs.

- RoD II: RoD for Fast Calls (Fig. 6).

Steps RoD II.1 and 2: When a fast call attempt $C_{\mathrm{f}}$ is newly generated at or handed off to the $i$ th microcell, the HCN first tries to assign a channel in the macrocell that is overlaid with the $i$ th microcell (following Criterion 2) to the call attempt. If the call attempt $C_{\mathrm{f}}$ is assigned a channel, the RoD procedure exists.

Step RoD II.3: If no idle macrocell channel is found in Step RoD II.1, then following Criterion 1, the HCN checks if there is any slow Mto-m repacking candidate. If so, the procedure proceeds to Step RoD II.4. Otherwise, Step II.6 is executed.

Steps RoD II.4 and 5: The HCN performs Mto-m repacking to generate a free macrocell channel for $C_{\mathrm{f}}$ and the procedure exits.

Steps RoD II.6 and 7: If no slow M-to-m repacking candidate is found, the HCN tries to allocate a channel in the $i$ th microcell to the call.

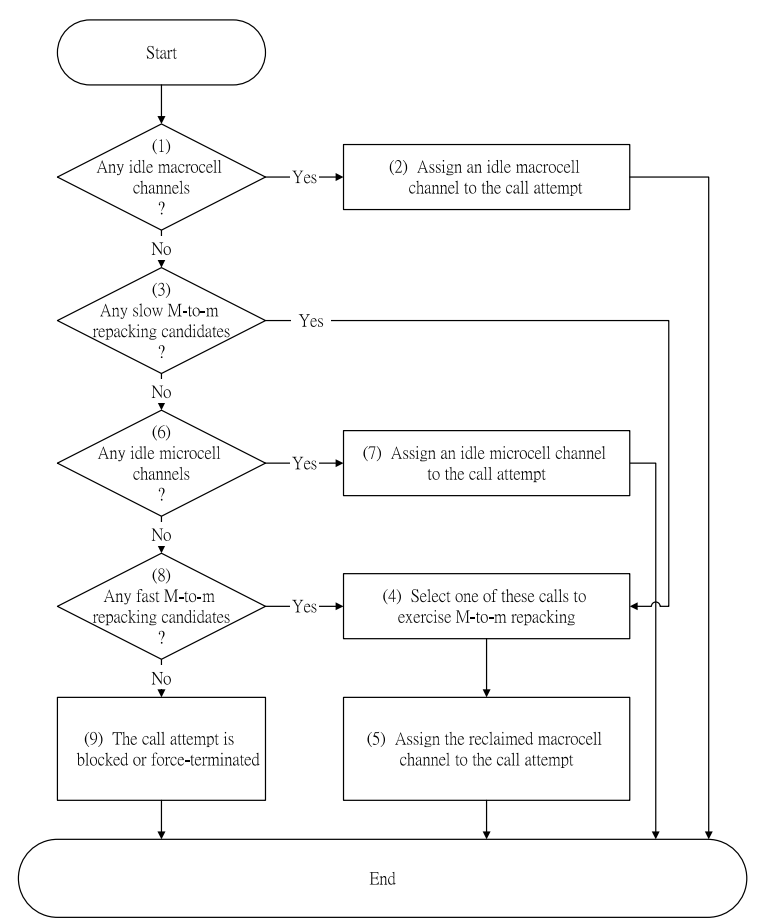

Fig. 6. RoD II: RoD for fast MSs.

Steps RoD II.8 and 9: If no idle channel is available in the microcell, the HCN checks if there is any fast M-to-m repacking candidate. If so, go to Step RoD II.4. Otherwise, the call attempt $C_{\mathrm{f}}$ is rejected.

In RoD, there are two alternatives to select the M-to-m repacking candidate at Steps RoD I.3 and RoD II.4. Random RoD (RoD-R) randomly selects a repacking candidate with the same probability. Load Balancing RoD (RoD-L) selects the repacking candidate whose microcell has the least traffic load. Both RoD-R and RoD-L can be adopted by an HCN that utilizes radio systems such as GSM/PCS1900 [13] or WCDMA [6], where the handoff decision is made by the network.

Partial RoD (PRoD; or preemption in $[19,20]$ ) is a special case of RoD, where Steps RoD I.5 (for slow calls) and RoD II.8 (for fast calls) are not executed. That is, PRoD only repacks slow calls.

Note that for both PRoD and RoD, Criterion 2 is used in channel assignment, but not in 
repacking. Only AR uses Criterion 2 in repacking. The primary purpose is to reduce the number of force-terminations for fast calls. Our study in Section 4 indicates that AR does not achieve its goal to reduce the force-termination probability as compared with RoD.

\section{System model for HCN channel assignment}

This section describes the input parameters and output measures for the HCN channel assignment model. For the demonstration purpose, we consider a wrapped mesh cell configuration as shown in Fig. 7. This configuration consists of four macrocells. Each macrocell covers $4 \times 4$ microcells. The wrapped topology simulates unbounded HCN so that the boundary cell effects can be ignored [12]. Without loss of generality, the MS moves to one of the four neighbor microcells with the same

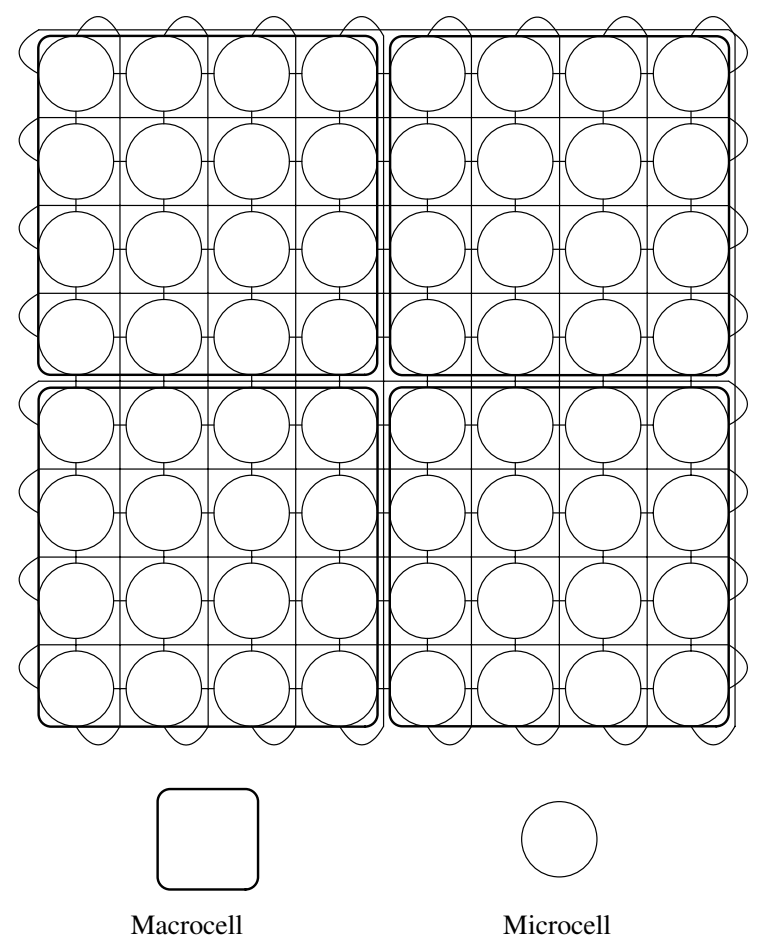

Fig. 7. Hierarchical cellular network with wrapped mesh configuration. probability (i.e., 0.25). Three types of input parameters are considered.

- System parameters: Each macrocell has $C$ radio channels, and each microcell has $c$ radio channels.

- Traffic parameters: The call arrivals to a microcell (for both incoming and outgoing calls) form a Poisson stream with rate $\lambda$. For fast MSs and slow MSs, the call arrivals rates are $f \lambda$ and $(1-f) \lambda$, respectively, where $0 \leqslant f \leqslant 1$. The call holding times have a Gamma distribution with mean $1 / \mu$ and variance $V_{\mathrm{c}}$ (the typical value for $1 / \mu$ is $1 \mathrm{~min}$ ).

- Mobility parameters: The microcell residence times of slow (fast) MSs have a Gamma distribution with mean $1 / \eta_{\mathrm{s}}\left(1 / \eta_{\mathrm{f}}\right)$ and variance $V_{\mathrm{m}, \mathrm{s}}$ $\left(V_{\mathrm{m}, \mathrm{f}}\right)$.

The Gamma distribution is often used in mobile telecommunications network modeling (call blocking analysis for PCS networks under general cell residence time [2], teletraffic analysis and mobility modeling for PCS network [3], analytical results for optimal choice of location update interval for mobility database failure restoration in PCS networks [4]). It has been shown that the distribution of any positive random variable can be approximated by a mixture of Gamma distributions (see Lemma 3.9 in reversibility and stochastic networks [9]). Several output measures are defined in this study:

$P_{\mathrm{b}}: \quad$ the probability that a new call is blocked.

$P_{\mathrm{f}}$ : the probability that a successfully connected call is forced to terminate because of handoff failure.

$P_{\mathrm{ff}}$ : the probability that a successfully connected fast call is forced to terminate because of handoff failure.

$P_{\text {nc }}: \quad$ the probability that a new call is blocked or a connected call is forced to terminate

$H$ : the expected number of handoffs (including repackings) occurred during a call.

Fig. 8 shows five types of handoffs. Handoff measures for these handoff types are defined as follows. 
$H_{\mathrm{mm}}$ : the expected number of handoffs from a microcell to another microcell during a call (Fig. 8(a)).

$H_{\mathrm{mM}}$ : the expected number of handoffs from a microcell to a macrocell during a call (Fig. 8(b)).

$H_{\mathrm{Mm}}$ : the expected number of handoffs from a macrocell to a microcell during a call (Fig. 8(c)).

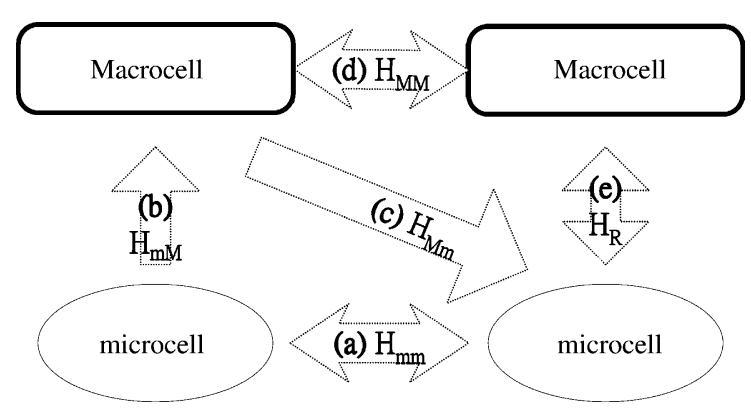

Fig. 8. Handoff types.
$H_{\mathrm{MM}}$ : the expected number of handoffs from a macrocell to another macrocell during a call (Fig. 8(d)).

$H_{\mathrm{R}}$ : the expected number of repackings (including $\mathrm{m}$-to-M and $\mathrm{M}$-to-m repackings) during a call (Fig. 8(e)).

From the above description, $H$ can be expressed as

$H=H_{\mathrm{mm}}+H_{\mathrm{mM}}+H_{\mathrm{Mm}}+H_{\mathrm{MM}}+H_{\mathrm{R}}$.

Based on above discussions, a discrete event simulation model for RoD-R is described in Appendix A. Other strategies (such as NR, AR, PRoD and RoD-L) can be studied by similar simulation models, and the details are omitted. Table 1 lists the notation described in this section, which will be used in the remainder of this paper. Note that the default values for the input parameters are also presented in Table 1.

Table 1

Notation

\begin{tabular}{|c|c|c|}
\hline Notation & Description & Default value for input parameter \\
\hline$C$ & The number of radio channels in a macrocell & 8 \\
\hline$c$ & The number of radio channels in a microcell & 10 \\
\hline \multicolumn{3}{|c|}{ Traffic parameters } \\
\hline$\lambda$ & The call arrival rate to a microcell & $7 \mu$ \\
\hline$f$ & The proportion of fasts calls & $9 \%$ \\
\hline $1 / \mu$ & The expected call holding time & $1 \mathrm{~min}$ \\
\hline$V_{\mathrm{c}}$ & The variance for call holding times & $1 / \mu^{2}$ \\
\hline \multicolumn{3}{|c|}{ Mobility parameters } \\
\hline $1 / \eta_{\mathrm{s}}$ & The expected microcell residence time of slow MSs & $10 / \mu$ \\
\hline $1 / \eta_{\mathrm{f}}$ & The expected microcell residence time of fast MSs & $1 / 5 \eta_{\mathrm{s}}$ \\
\hline$V_{\mathrm{m}, \mathrm{s}}$ & The variance for microcell residence times of slow MSs & $100 / \mu^{2}$ \\
\hline$V_{\mathrm{m}, \mathrm{f}}$ & The variance for microcell residence times of fast MSs & $V_{\mathrm{m}, \mathrm{s}} / 25$ \\
\hline \multicolumn{3}{|c|}{ Output measures } \\
\hline$P_{\mathrm{b}}$ & The call blocking probability & \\
\hline$P_{\mathrm{f}}$ & The force-termination probability & \\
\hline$P_{\mathrm{ff}}$ & The force-termination probability of fast calls & \\
\hline$P_{\mathrm{nc}}$ & The call incomplete probability & \\
\hline$H_{\mathrm{mm}}$ & The expected number of microcell-to-microcell handoffs per call & \\
\hline$H_{\mathrm{mM}}$ & The expected number of microcell-to-macrocell handoffs per call & \\
\hline$H_{\mathrm{Mm}}$ & The expected number of macrocell-to-microcell handoffs per call & \\
\hline$H_{\mathrm{MM}}$ & The expected number of macrocell-to-macrocell handoffs per call & \\
\hline$H_{\mathrm{R}}$ & The expected number of repackings & \\
\hline & (including $\mathrm{m}$-to-M and $\mathrm{M}$-to-m repackings) during a call & \\
\hline$H$ & The expected number of handoffs and repackings per call & \\
\hline
\end{tabular}




\section{Results and discussions}

We compare NR, AR, PRoD, RoD-R and RoD-L in terms of the output measures listed in Table 1. In our numerical examples, the radio channel number is $c=10$ for every microcell and the expected microcell residence time for slow MSs is 5 times the value for fast MSs (i.e., 1/ $\left.\eta_{\mathrm{s}}=5 / \eta_{\mathrm{f}}\right)$. The effects of the input parameters are described as follows.

Effect of the macrocell channel number $C$. Fig. 9 (a)-(d) plot $P_{\mathrm{ff}}, P_{\mathrm{b}}, P_{\mathrm{f}}$ and $P_{\mathrm{nc}}$ as functions of $C$,
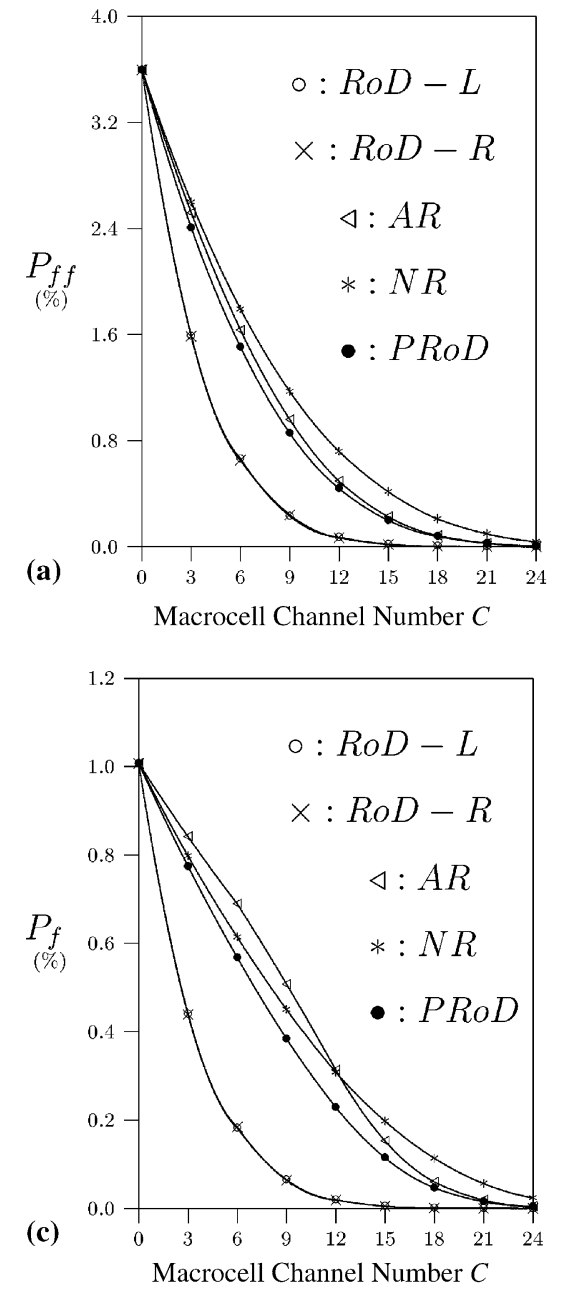

where the values for the input parameters except $C$ follow the default values listed in Table 1 . These figures show an intuitive result that for all approaches, $P_{\mathrm{ff}}, P_{\mathrm{b}}, P_{\mathrm{f}}$ and $P_{\mathrm{nc}}$ decrease as $C$ increases. We also observe that the $P_{\mathrm{ff}}, P_{\mathrm{b}}, P_{\mathrm{f}}$ and $P_{\text {nc }}$ are more sensitive to the change of $C$ for small $C$ values than for large $C$ values. Since the macrocell channels are the bottleneck resources when $C$ is small, increasing $C$ significantly reduces $P_{\mathrm{ff}}, P_{\mathrm{b}}$, $P_{\mathrm{f}}$ and $P_{\mathrm{nc}}$. Fig. 10(a)-(d) plot $H_{\mathrm{mm}}, H_{\mathrm{mM}}, H_{\mathrm{R}}$ and $H$ as functions of $C$, where the values for the input parameters except $C$ follow the default values listed in Table 1. Fig. 10(a) shows that for
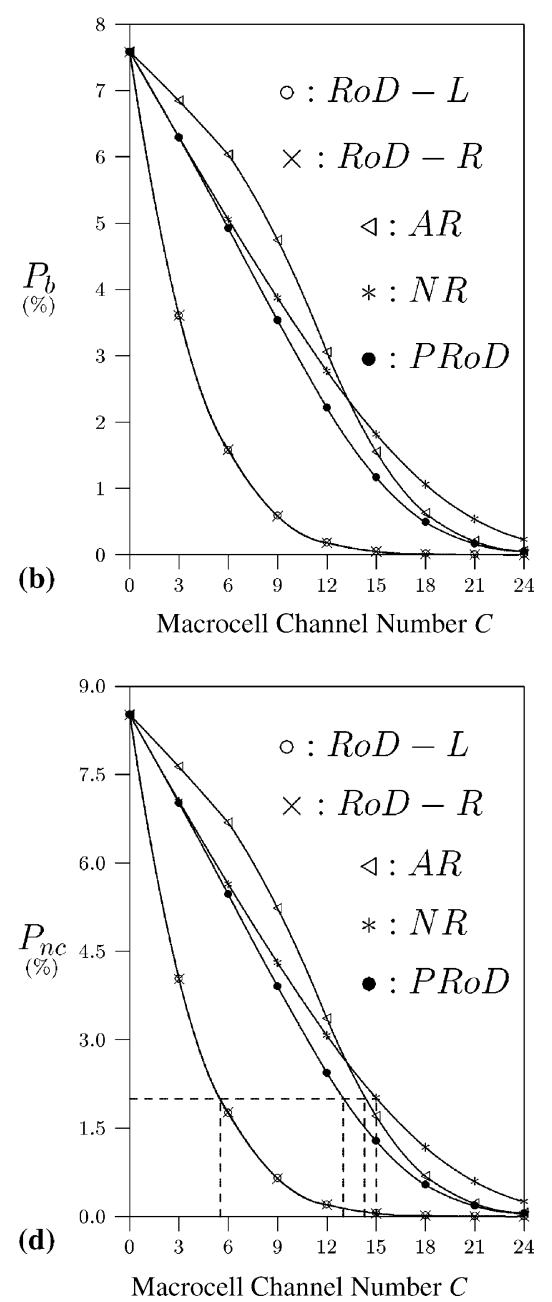

Fig. 9. Effects of macrocell channel number $C$ on (a) force-termination probability $P_{\mathrm{ff}}$ of fast calls, (b) new call blocking probability $P_{\mathrm{b}}$, (c) force-termination probability $P_{\mathrm{f}}$ and (d) incomplete probability $P_{\mathrm{nc}}$. 

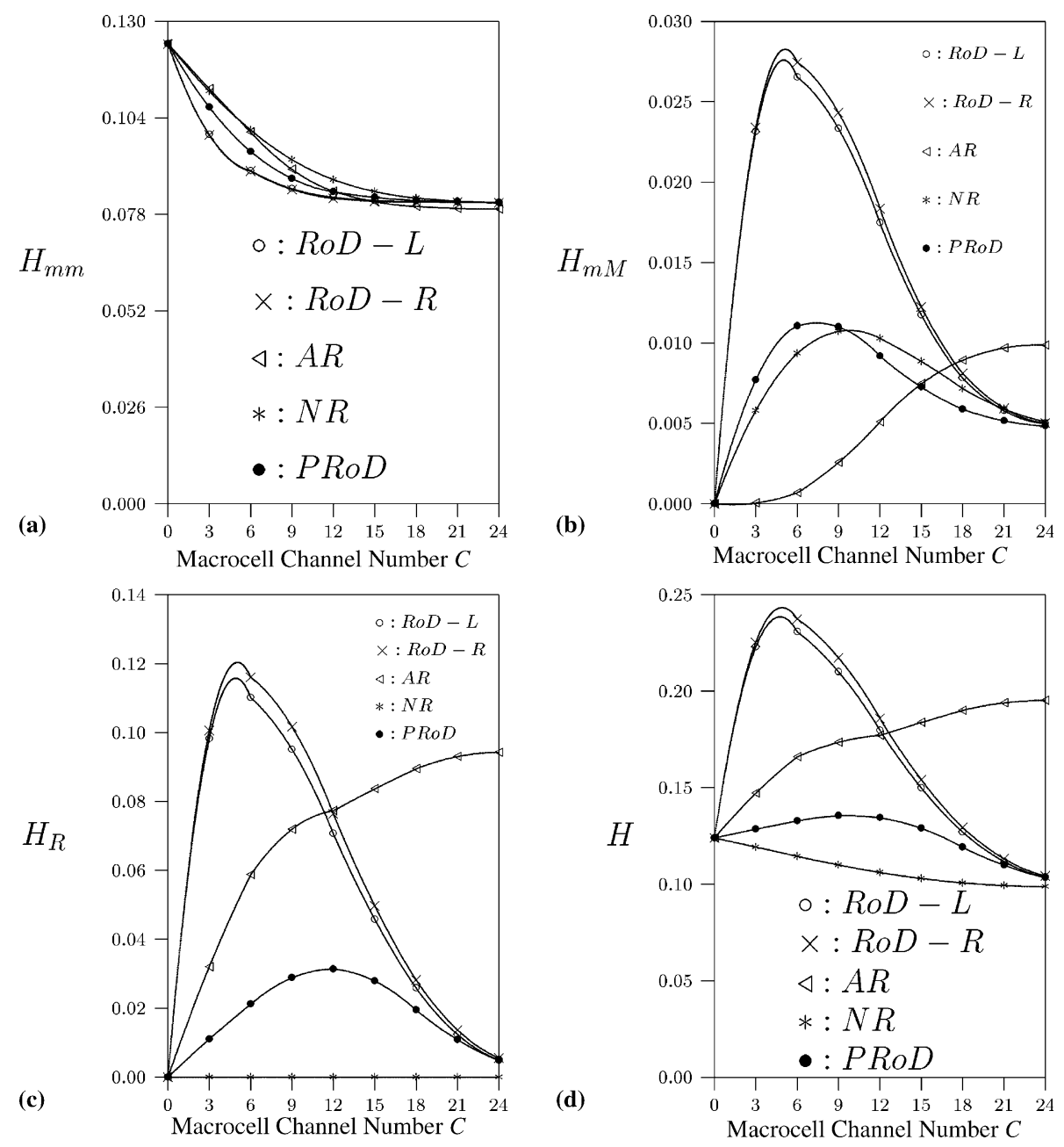

Fig. 10. Effects of the macrocell channel number $C$ on (a) expected number $H_{\mathrm{mm}}$ of microcell to microcell handoffs, (b) expected number $H_{\mathrm{mM}}$ of microcell to macrocell handoffs, (c) expected number $H_{\mathrm{R}}$ of repackings and (d) expected number $H$ of handoffs.

all approaches, $H_{\mathrm{mm}}$ is a decreasing function of $C$. This phenomenon is due to the fact that when $C$ increases, more calls (especially fast calls) will occupy macrocell channels, and the number of microcell to microcell handoffs will decrease. Fig. 10(b) shows that for $\mathrm{AR}, H_{\mathrm{mM}}$ is an increasing function of $C$. Increasing $C$ results in more idle macrocell channels, and more calls (especially slow calls) will be handed off from microcells to macrocells. For NR, PRoD and RoD, $H_{\mathrm{mM}}$ increases and then decreases as $C$ increases. When $C$ is small, macrocell channels are the bottleneck resources and increasing $C$ results in more calls handed off from microcells to macrocells (especially fast calls that overflow to microcells). When $C$ is large ( $C>5$ in Fig. 10(b)), macrocell channels are no longer the bottleneck resources and less fast calls overflow to microcells. In this case, increasing $C$ results in decreasing of $H_{\mathrm{mM}}$. The performance figures for $H_{\mathrm{Mm}}$ and $H_{\mathrm{MM}}$ are similar to that for $H_{\mathrm{mM}}$, and the details are omitted. Fig. 10(c) shows that for $\mathrm{AR}, H_{\mathrm{R}}$ increases as $C$ increases. Increasing $C$ results in more slow calls that would overflow to macrocells, and thus more calls are repacked. On the other hand, for PRoD and RoD, $H_{\mathrm{R}}$ increases and then decreases as $C$ 
increases. This non-trivial phenomenon is explained as follows. When $C$ is small $(C<5$ for RoD and $C<12$ for PRoD in Fig. 10(c)), increasing $C$ results in more M-to-m repacking candidates, and more on-demand repackings are exercised. When $C$ is large, macrocell channels are no longer the bottleneck resources. Increasing $C$ results in less blockings as well as force-terminations, and less on-demand repackings are needed. Therefore $H_{\mathrm{R}}$ decreases as $C$ increases in this case. Fig. 10(d) shows the net effects of repackings and all types of handoffs. In this figure, as $C$ increases, $H$ decreases for NR and increases for AR. For PRoD and RoD, $H$ increases and then decreases as $C$ increases.

Comparison of NR, AR, PRoD, RoD-R and RoDL. Fig. 9(a)-(d) compare NR, AR, PRoD, RoD$\mathrm{R}$ and RoD-L on $P_{\mathrm{ff}}, P_{\mathrm{b}}, P_{\mathrm{f}}$ and $P_{\mathrm{nc}}$, respectively. These figures show that RoD-R and RoD-L have smaller $P_{\mathrm{ff}}, P_{\mathrm{b}}, P_{\mathrm{f}}$ and $P_{\mathrm{nc}}$ values than NR, AR and PRoD. Furthermore, AR has higher $P_{\mathrm{nc}}$ than NR when $C$ is small, and the opposite result is observed when $C$ is large $(C>12.5$ in Fig. 9(d)). This non-trivial phenomenon is explained as follows. Consider the case when $C$ is much less than the number of fast calls. In AR, m-to-M repacking is always exercised, and therefore macrocells have less idle channels in AR than in NR. In this case, the $P_{\mathrm{nc}}$ value is higher for AR than for NR. On the other hand, when $C$ is large, the effect of M- to-m repacking for slow calls becomes more significant. Thus macrocells have more idle channels in AR than in NR. Specifically, if the HCN is engineered at $P_{\mathrm{nc}}=2 \%$ (see the horizontal dashed line in Fig. 9(d)), $C=5.5$ for RoD, $C=13$ for PRoD, $C=14.5$ for $\mathrm{AR}$, and $C=15$ for NR. Thus RoD can save at least 7 macrocell channels over other approaches.

Fig. 10 (c) shows that $H_{\mathrm{R}, \mathrm{RoD}-\mathrm{R}}>H_{\mathrm{R}, \mathrm{RoD}-\mathrm{L}}>$ $H_{\mathrm{R}, \mathrm{AR}}>H_{\mathrm{R}, \mathrm{PRoD}}>H_{\mathrm{R}, \mathrm{NR}}=0$ when $C$ is small $(C<12)$. When $C$ is large $(C>12), H_{\mathrm{R}, \mathrm{AR}}>$ $H_{\mathrm{R}, \mathrm{RoD}-\mathrm{R}}>H_{\mathrm{R}, \mathrm{RoD}-\mathrm{L}}>H_{\mathrm{R}, \mathrm{PRoD}}>H_{\mathrm{R}, \mathrm{NR}}=0$. Similar phenomena for $H$ are observed in Fig. 10(d). Although NR, AR and PRoD have smaller $H_{\mathrm{R}}$ and $H$ values than RoD, the increase of $H_{\mathrm{R}}$ and $H$ does not results in the increase of $P_{\mathrm{ff}}, P_{\mathrm{b}}$, $P_{\mathrm{f}}$ and $P_{\mathrm{nc}}$ in RoD (see Fig. 9(a)-(d)).

Effect of the proportion $f$ of fast MSs. Fig. 11 plots $P_{\mathrm{nc}}$ as a function of $f$, where the values for the input parameters except $f$ follow the default values listed in Table 1. In the figure, the call arrival rates for fast and slow MSs are $f \lambda$ and $(1-f) \lambda$, respectively. This figure shows that for all approaches, $P_{\text {nc }}$ increases as $f$ increases. Increasing of fast calls results in the increase of handoffs and hence forceterminations. This figure also shows that RoD approaches (i.e., RoD-R and RoD-L) are less sensitive to $f$ than other approaches. Furthermore, when $f$ is large $(f>8 \%$ for $\mathrm{AR}$ and $f>18 \%$ for $\mathrm{PRoD}$ ), $\mathrm{AR}$ and $\mathrm{PRoD}$ have higher

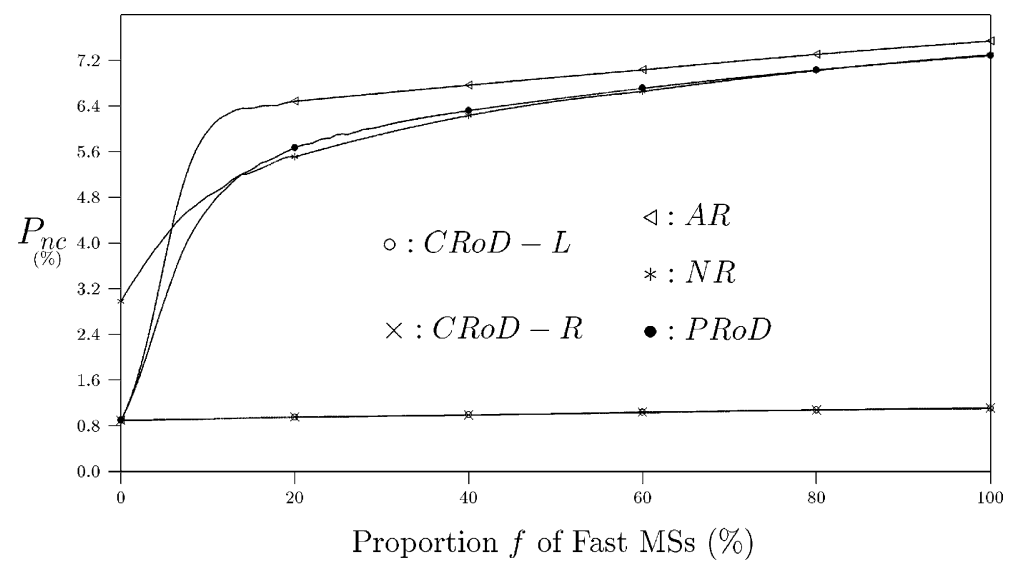

Fig. 11. Effects of the proportion $f$ of fast MSs on incomplete probability $P_{\mathrm{nc}}$. 
$P_{\mathrm{nc}}$ values than NR. The reason is that for AR, when $f$ is large, the effect of m-to-M repackings is more significant than that of M-to-m repackings. Thus, less idle channels are available in macrocells for AR than for NR, and more calls are blocked or forced to terminate in AR. For PRoD, when $f$ is large, more fast calls repack slow calls, which occupy more macrocell channels. Therefore, more slow calls are blocked or forced to terminate.

Effect of the MS mobility (i.e., mean microcell residence times). Fig. 12 plots $P_{\mathrm{nc}}$ as a function of the microcell mobility rates (i.e., $\eta_{\mathrm{s}}$ for slow MSs and $\eta_{\mathrm{f}}=5 \eta_{\mathrm{s}}$ for fast MSs), where the values for the input parameters except $1 / \eta_{\mathrm{s}}$ and $1 / \eta_{\mathrm{f}}$ follow the default values listed in Table 1. This figure shows that $P_{\mathrm{nc}}$ increases as $\eta_{\mathrm{s}}$ increases. This figure also shows that to keep the same $P_{\mathrm{nc}}$ performance, (e.g., $P_{\mathrm{nc}}=7 \%$ ), RoD can support much faster MSs (at least $2^{3}$ times the $\eta_{\mathrm{s}}$ value) than other approaches.

Effect of the arrival rate $\lambda$. Fig. 13 plots $P_{\mathrm{nc}}$ as a function of $\lambda$, where the values for the input parameters except $\lambda$ follow the default values listed in Table 1. This figure shows that $P_{\mathrm{nc}}$ increases as $\lambda$ increases. It also shows that to keep the same $P_{\mathrm{nc}}$ performance (e.g., $P_{\mathrm{nc}}=2 \%$ ), RoD can support more call arrivals (at least 18\%) than other approaches.

Effect of the variance $V_{c}$ for the call holding times. Fig. 14 plots $P_{\mathrm{nc}}$ and $H$ as functions of $V_{\mathrm{c}}$, where the values for the input parameters except $V_{\mathrm{c}}$ follow the default values listed in Table 1. Fig. 14 shows that $P_{\mathrm{nc}}$ and $H$ decrease as $V_{\mathrm{c}}$ increases. Note that, for the call holding time distributions with the same mean value $1 / \mu$, the standard deviation $\sigma=\sqrt{V_{c}}$. By the Chebyshev's Inequality, the probability that the call holding times are out of range $\left[1 / \mu-\left(5 \sqrt{V_{\mathrm{c}}}\right) / 3,1 / \mu+\left(5 \sqrt{V_{\mathrm{c}}}\right) / 3\right]$ is smaller than $36 \%$ for all $V_{\mathrm{c}}$ values. For example, if $V_{\mathrm{c}}=100 / \mu^{2}$, then $\left(5 \sqrt{V_{\mathrm{c}}}\right) / 3=50 / 3 \mu$ and the probability that the call holding time exceeds $53 /$ $3 \mu$ is smaller than $36 \%$. As $V_{\mathrm{c}}$ increases, more long and short call holding times are observed. More short call holding times implies that more calls are completed before next new call attempts arrive or next handoff attempts are exercised. Thus the numbers of blocked calls, force-terminated calls and handoffs decrease.

Effect of the variances for the microcell residence times. Fig. 15 plots $P_{\mathrm{nc}}$ and $H$ as functions of variances (i.e., $V_{\mathrm{m}, \mathrm{s}}$ for slow MSs and $V_{\mathrm{m}, \mathrm{f}}=V_{\mathrm{m}, \mathrm{s}} / 25$

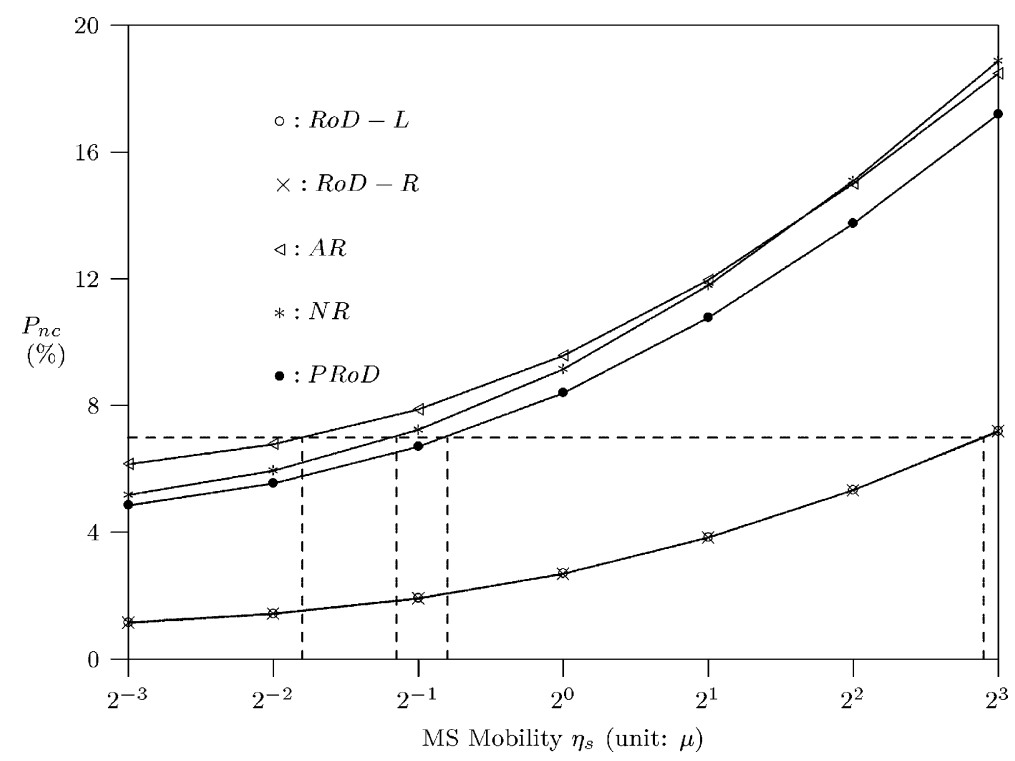

Fig. 12. Effects of MS mobility on incomplete probability $P_{\text {nc }}$. 


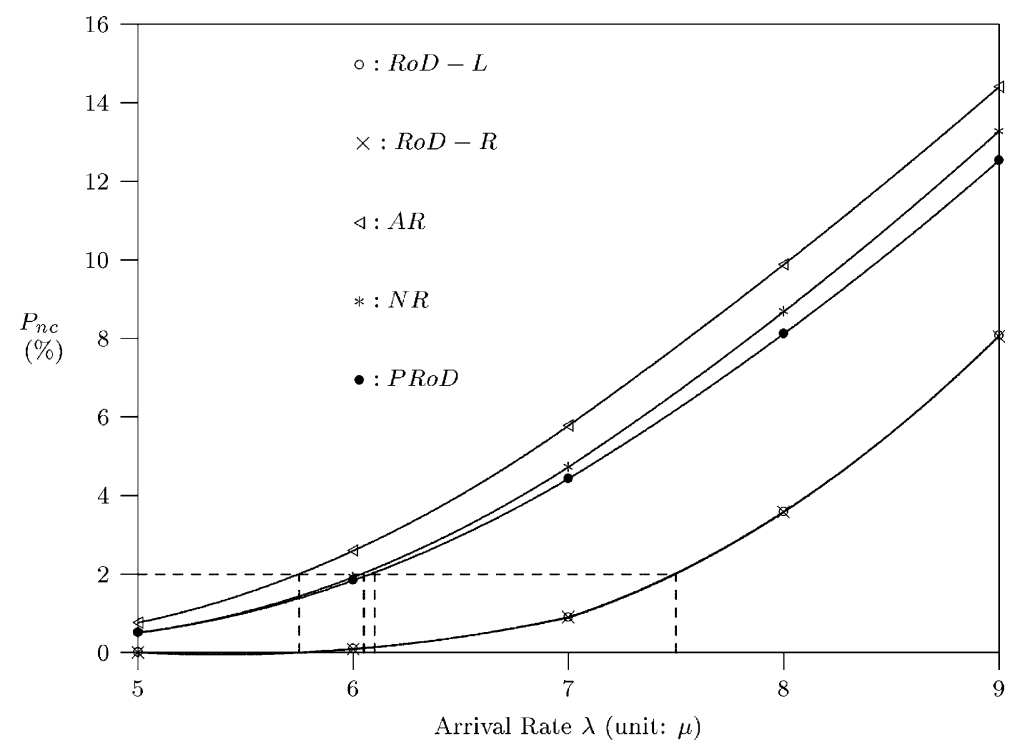

Fig. 13. Effects of the arrival rate $\lambda$ on incomplete probability $P_{\text {nc. }}$.
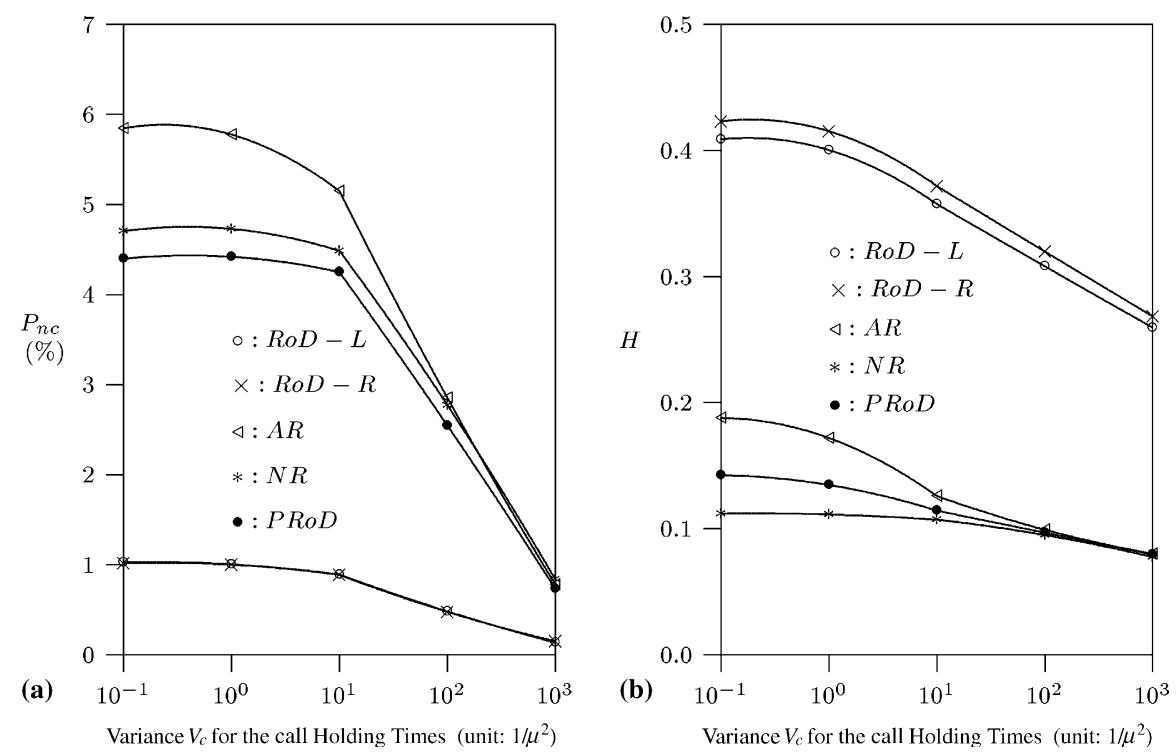

Fig. 14. Effects of the variance $V_{\mathrm{c}}$ for the call holding times on (a) incomplete probability $P_{\mathrm{nc}}$ and (b) expected number $H$ of handoffs.

for fast MSs) for the microcell residence times, where the values for the input parameters except $V_{\mathrm{m}, \mathrm{s}}$ and $V_{\mathrm{m}, \mathrm{f}}$ follow the default values listed in Table 1. Fig. 15 shows that $P_{\mathrm{nc}}$ and $H$ decrease as $V_{\mathrm{m}, \mathrm{s}}$ increases. From the residual life theorem
[10], the mean value of the first microcell residence time increases as $V_{\mathrm{m}, \mathrm{s}}$ increases, which implies that more calls will complete in the first microcell before they are handed off to the next cells. Therefore, both $P_{\mathrm{nc}}$ and $H$ drop as $V_{\mathrm{m}, \mathrm{s}}$ increases. 

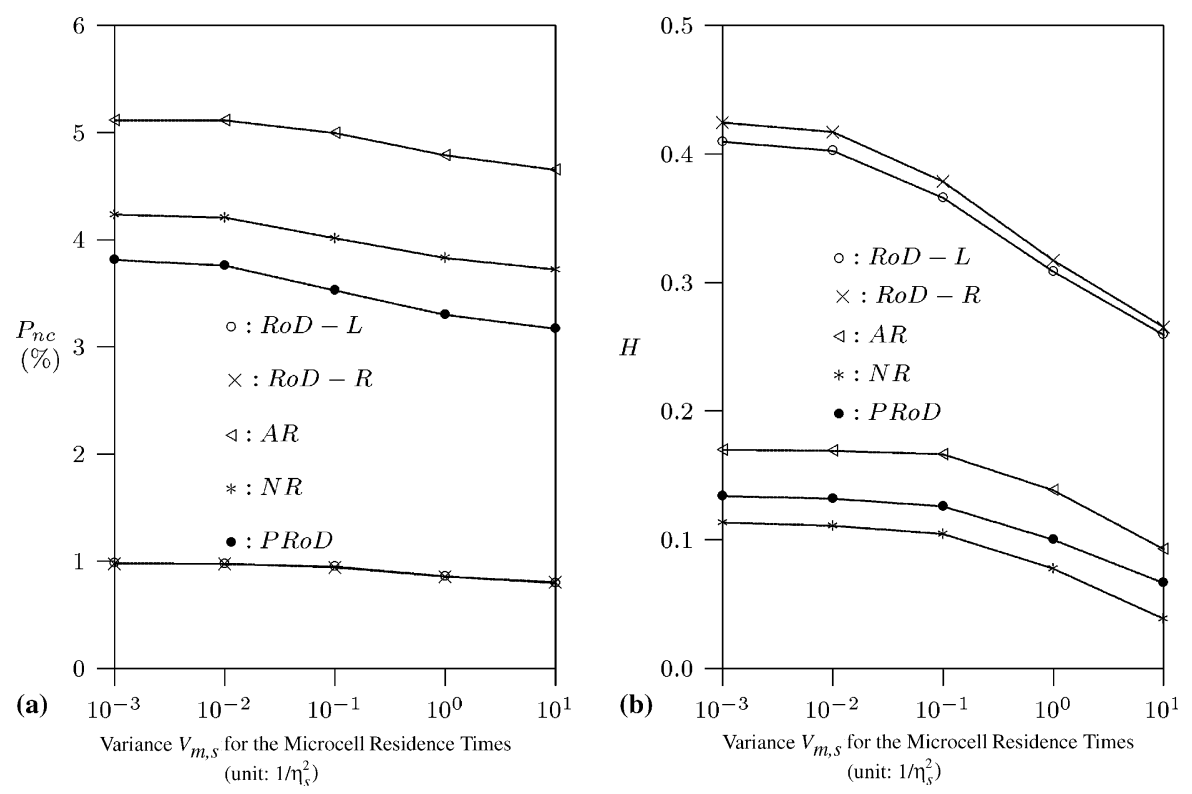

Fig. 15. Effects of variance $V_{\mathrm{m}, \mathrm{s}}$ for microcell residence times on (a) incomplete probability $P_{\mathrm{nc}}$ and (b) expected number $H$ of handoffs.

\section{Conclusions}

By considering the moving speeds of MSs, this paper proposed the repacking on demand (RoD) approach for channel assignment in the hierarchical cellular network $(\mathrm{HCN})$. We developed simulation models to investigate the RoD performance on the blocking probability $P_{\mathrm{b}}$, the force-termination probability $P_{\mathrm{f}}$, the incomplete probability $P_{\mathrm{nc}}$ and the expected number of handoffs $H$ during a call (for both slow and fast calls). We compared RoD with other HCN channel assignment approaches including No Repacking (NR), Always Repacking (AR) and Partial RoD (PRoD). Our study indicated that

- If the requests for the macrocell channels can not be satisfied (e.g., when the number of the macrocell channel is small, the call arrival rate and MS mobility are high, and so on), macrocell channels are the bottleneck resources. In this case, RoD significantly reduces $P_{\mathrm{b}}, P_{\mathrm{f}}$, and $P_{\mathrm{nc}}$ as compared with other approaches.

- The $P_{\mathrm{b}}, P_{\mathrm{f}}$, and $P_{\mathrm{nc}}$ performance for RoD is not sensitive to the proportion of fast call arrivals as compared with other approaches. That is, the increase of fast calls does not affect RoD as much as other approaches.
- With the same $P_{\text {nc }}$ performance, RoD can support much faster MSs and/or more call arrivals than other approaches.

- In RoD, Random RoD (RoD-R) and Load Balancing RoD (RoD-L) have the same $P_{\mathrm{b}}$, $P_{\mathrm{f}}$, and $P_{\mathrm{nc}}$ performance. Note that in repacking, macrocell is a resource pool used to adjust traffic load of each microcell. That is, repacking already conducts the load balancing function to effectively balance the system workload, and the load-balancing improvement by RoD-L becomes insignificant. Therefore, the performance resulted from random selection for repacking candidates (i.e., RoD-R) is similar to that for RoD-L. This result is very important for network operators because RoD-R is much easier to implement than RoD-L.

\section{Acknowledgments}

We would like to thank the anonymous reviewers. Their comments have significantly improved the quality of this paper. This work was sponsored in part by Intel, Microsoft, National Science Council under contracts NSC93-2213-E-002-083, NSC932213-E-002-025 and NSC93-2213-E-009-100. 


\section{Appendix A. Simulation model for RoD-R}

This appendix describes the discrete event simulation model for RoD-R. In this model, three types of events are defined to represent call arrival, call completion, and MS movement. The following attributes are defined for an event e:

- The type attribute indicates the event type. An Arrival event represents a new call arrival. A Move event represents an MS movement from one cell to another. A Complete event represents a call completion.

- The ts attribute indicates the time when the event occurs.

- The tc attribute indicates the time when the call corresponding to e will complete. Note that tc $\geqslant$ ts.

- The mc attribute indicates the microcell where the MS (corresponding to this event) resides.

- The ma attribute indicates the macrocell where the MS (corresponding to this event) resides.

- The speed attribute indicates the moving speed (i.e., Slow or Fast) of the MS (corresponding to this event).

- The is_macro attribute indicates whether the call corresponding to this event occupies a macrocell channel. If a macrocell channel is occupied, is_macro $=1$. Otherwise, is_macro $=$ 0 .

In the simulation model, an array $m c_{-} c h[i]$ is used to represent the number of the idle channels of microcell $i$. Another array ma_ch[j] is used to represent the number of the idle channels of macrocell $j$. Two variables new_mc and new_ma are used to indicate the target micro and macro cells where the call (corresponding to current event) is newly generated from or handed off to. The output measures of the simulation are the number $N$ of total call arrivals during the simulation, the number $N_{\mathrm{b}}$ of blocked calls, the number $N_{\mathrm{f}}$ of force-terminated calls, the number $N_{\mathrm{R}}$ of repackings, the number $N_{\mathrm{h}}$ of handoffs, the number $N_{\mathrm{sf}}$ of successfully connected fast calls, and the number $N_{\mathrm{ff}}$ of force-terminated fast calls. From the above output measures, we can compute

$$
\begin{aligned}
& P_{\mathrm{b}}=\frac{N_{\mathrm{b}}}{N}, \quad P_{\mathrm{f}}=\frac{N_{\mathrm{f}}}{N-N_{\mathrm{b}}}, \quad P_{\mathrm{nc}}=\frac{N_{\mathrm{b}}+N_{\mathrm{f}}}{N}, \\
& P_{\mathrm{ff}}=\frac{N_{\mathrm{ff}}}{N_{\mathrm{sf}}}, \quad H_{\mathrm{R}}=\frac{N_{\mathrm{R}}}{N-N_{\mathrm{b}}} \quad \text { and } \quad H=\frac{N_{\mathrm{h}}}{N-N_{\mathrm{b}}} .
\end{aligned}
$$

A simulation clock is maintained to indicate the simulation progress, which is the timestamp of the event being processed. All events are inserted into the event list, and are deleted/processed from the event list in the non-decreasing timestamp order. Figs. 16 and 17 show the simulation flow chart for RoD-R. In this flow chart, Step 1.1 initializes the input parameters. Step 1.2 generates the first Arrival events for each microcell and inserts these events into the event list. In Steps 1.3 and 1.4, the next event $\mathbf{e}$ in the event list is processed based on its type. There are three cases:

Case I. e.type $=$ Arrival: At Step 1.5, if $N-N_{\mathrm{b}}>N^{*}\left(\right.$ in our simulation, $N^{*}=6 \times 10^{5} \times$ 64), then Step 1.6 computes the output measures using (2), and the simulation terminates. Otherwise, Step 1.7 generates the next Arrival event $\mathbf{e}_{1}$ for the same microcell (i.e., $\mathbf{e}_{\mathbf{1}} \cdot \mathbf{m c}=\mathbf{e} . \mathbf{m c}$, $\mathbf{e}_{1} \cdot \mathbf{m a}=\mathbf{e}$.ma,$\quad \mathbf{e}_{1}$.speed $=$ e.speed and $\mathbf{e}_{1}$.is_macro $=0$ ), and sets the target cell where the incoming call arrives (new_mc $=\mathbf{e} . \mathbf{m c}$ and $n e w \_m a=$ e.ma). The timestamp of $\mathbf{e}_{\mathbf{1}}$ equals e.ts plus the inter call arrival time generated by a random number generator. Then $\mathbf{e}_{\mathbf{1}}$ is inserted into the event list, and the simulation proceeds to execute Algorithm $A$ in Fig. 17.

In Algorithm $A$ (see Fig. 17), the $\mathrm{HCN}$ tries to allocate a channel for the call. The steps are described as follows. If e.speed $=$ Slow at Step 2.1, Step 2.2 checks if microcell new_mc has idle channels (i.e., $m c \_c h\left[n e w \_m c\right]>0$ ). If so, a channel is assigned to the incoming call. Then $m c_{-} c h\left[n e w \_m c\right]$ is decremented by 1 at Step 2.3 and this algorithm terminates at exit B (i.e., the call is assigned a channel). At Step 2.2, if microcell new_mc has no idle channel (i.e., $m c \_c h\left[n e w \_m c\right]=0$ ), the call attempt overflows to macrocell new_ma. Then Step 2.4 checks if macrocell new_ma has idle channels (i.e., $\left.m a \_c h\left[n e w \_m a\right]>0\right)$. If so, a macrocell channel is assigned to the call at Step 2.5. In this case, ma_ch-[new_ma] is decremented by 1 , and 


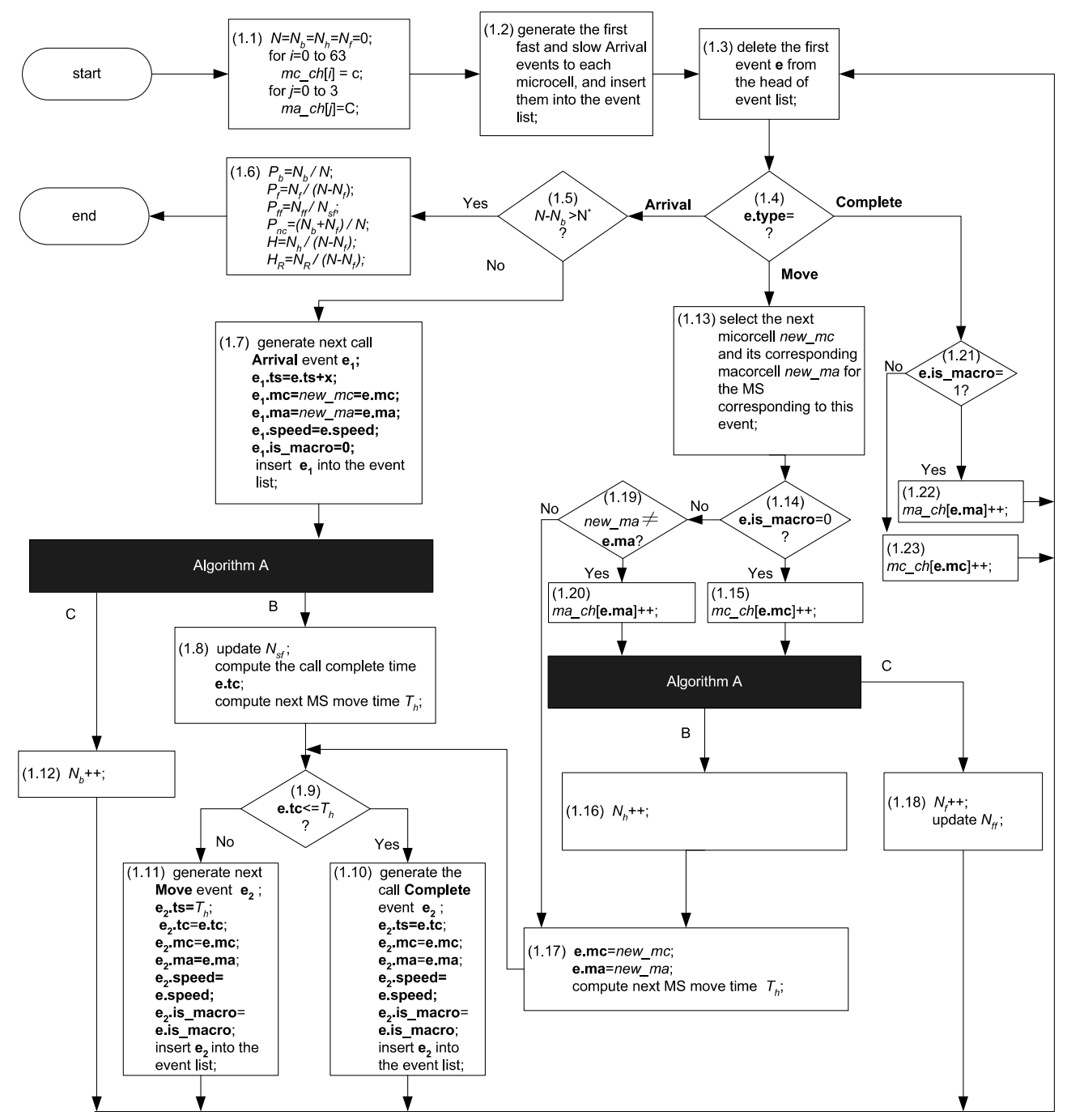

Fig. 16. Simulation flow chart for RoD-R.

e.is_macro is set to 1 . Then this algorithm terminates at exit $\mathrm{B}$. If macrocell new_ma has no idle channel (i.e., $m a \_c h\left[n e w \_m a\right]=0$ ) at Step 2.4, then Step 2.6 checks if there is any slow M-to-m repacking candidate in macrocell new_ma. If so, Step 2.7 randomly selects a slow M-to-m repacking candidate (i.e., a call corresponding to a Complete or Move event $\mathbf{e}_{3}$ ) to be handed off from macrocell $n e w \_m a$ to microcell $\mathbf{e}_{3}$.mc (i.e., $\mathbf{e}_{3}$.is_macro is set to 0 ), and the reclaimed macrocell channel is assigned to the incoming call (i.e., e.is_macro is set to 1). Step 2.7 also decrements $m c_{-} c h\left[\mathbf{e}_{3} . \mathbf{m c}\right]$ by
1 , and increments both $N_{\mathrm{R}}$ and $N_{\mathrm{h}}$ by 1 . Then this algorithm terminates at exit B. If no slow M-to-m repacking candidate is found at Step 2.6, Step 2.8 checks if there is any fast M-to-m repacking candidate in macrocell new_ma. If so, Step 2.7 is executed to randomly select a fast M-to-m repacking candidate, and this algorithm terminates at exit B. Otherwise, if no fast M-to-m repacking candidate is found at Step 2.8, the incoming call is not assigned any idle channel. In this case, this algorithm terminates at exit $\mathrm{C}$ (i.e., no channel is assigned to the call). 


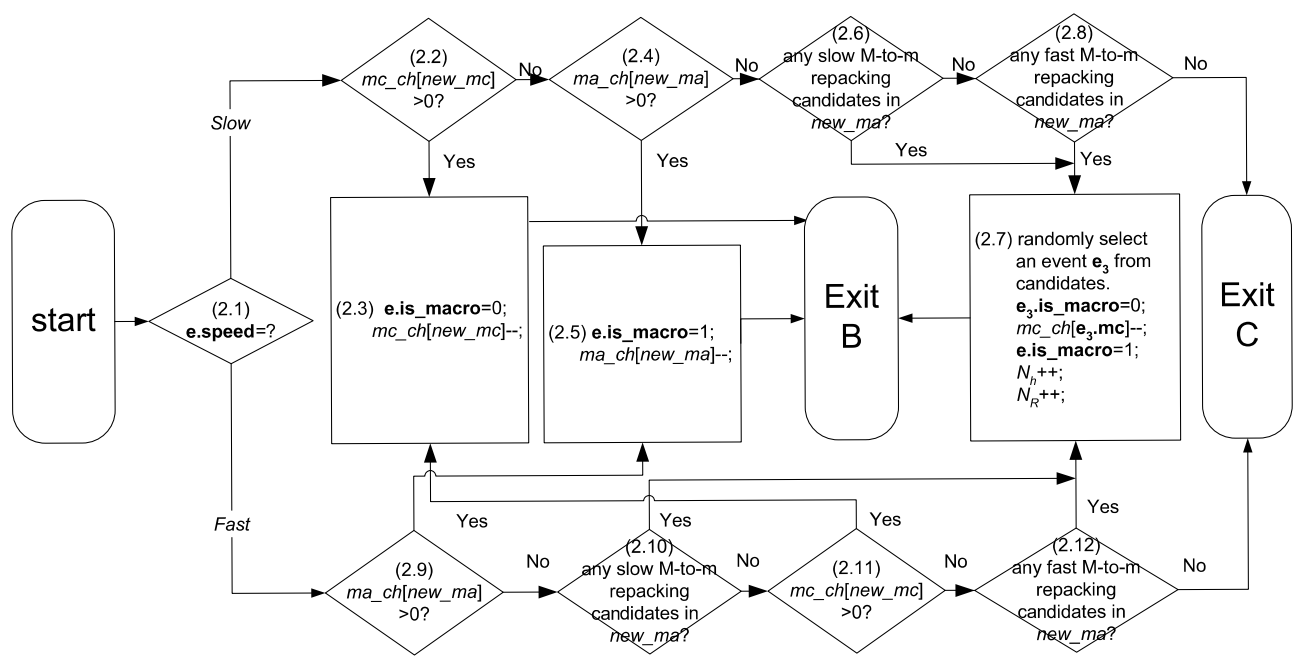

Fig. 17. Flow chart of algorithm A.

For the case of e.speed $=$ Fast at Step 2.1, Step 2.9 checks if macrocell new_ma has idle channels (i.e., $m a \_c h\left[n e w \_m a\right]>0$ ). If so, Step 2.5 is executed, and the algorithm terminates at exit B. Otherwise, Step 2.10 checks if there is any slow M-to-m repacking candidate in macrocell new_ma. If so, Step 2.7 is executed, and this algorithm terminates at exit B. If no slow M-to-m repacking candidates are found in macrocell new_ma, then Step 2.11 checks if microcell new_mc has idle channels (i.e., $m c_{-} c h\left[n e w \_m c\right]>0$ ). If so, Step 2.3 is executed, and this algorithm terminates at exit $\mathrm{B}$. If microcell new_mc has no idle channel at Step 2.11, Step 2.12 checks if there is any fast M-to-m repacking candidate in macrocell new_ma. If so, Step 2.7 is executed, and this algorithm terminates at exit B. Otherwise, the incoming call is not assigned any idle channel, and the this algorithm terminates at exit $\mathrm{C}$.

If any channel is assigned to the incoming call in Fig. 17 (i.e., Algorithm $A$ exits from B), Step 1.8 updates the $N_{\text {sf }}$ value (i.e., if this is a fast call, increment $N_{\text {sf }}$ by 1 ). Step 1.8 then computes the call completion time e.tc as e.ts plus the call holding time. Step 1.8 also determines the MS move time $T_{\mathrm{h}}$ when the MS moves out of the microcell. $T_{\mathrm{h}}$ equals e.ts plus the cell residence time. Then Step 1.9 determines the next event (i.e., a Move event or Complete event) for the call correspond- ing to e. If the MS will move to another cell after call completion (i.e., e.tc $\leqslant T_{\mathrm{h}}$ ), then Step 1.10 is executed to generate a Complete event $\mathbf{e}_{2}$ where $e_{2} \cdot$ ts $=$ e.tc $, \quad e_{2} \cdot m c=e . m c ， e_{2} \cdot m a=e . m a, \quad e_{2} \cdot$ spee$\mathbf{d}=\mathbf{e}$.speed and $\mathbf{e}_{2}$.is_macro $=$ e.is_macro. Event $\mathbf{e}_{\mathbf{2}}$ is inserted into the event list. Otherwise, if the MS moves to another cell before the call completion (i.e., e.tc $>T_{\mathrm{h}}$ ) at Step 1.9, then Step 1.11 is executed to generate the next Move event $\mathbf{e}_{2}$ with the timestamp $T_{\mathrm{h}}$ for this call (i.e., $\mathbf{e}_{2} . \mathbf{t s}=T_{\mathrm{h}}$, $\mathrm{e}_{2} \cdot \mathrm{tc}=\mathrm{e} . \mathrm{tc}, \mathrm{e}_{2} \cdot \mathrm{mc}=\mathrm{e} \cdot \mathrm{mc}, \mathrm{e}_{2} \cdot \mathrm{ma}=\mathrm{e} \cdot \mathrm{ma}, \mathrm{e}_{2} \cdot \mathrm{spee}-$ $\mathbf{d}=$ e.speed and $\mathbf{e}_{2}$.is_macro $=\mathbf{e}$.is_macro). Event $\mathbf{e}_{2}$ is inserted into the event list. On the other hand, if $\mathrm{HCN}$ has no idle channel for the incoming call (i.e., Algorithm $A$ exits from C), the incoming call is blocked and $N_{\mathrm{b}}$ is incremented by 1 at Step 1.12 .

Case II. e.type $=$ Move: Step 1.13 selects the next microcell new_mc and its macrocell new_ma for the MS corresponding to event e. At Step 1.14, if the call of the MS occupies a microcell channel (i.e., e.is_macro $=0$ ), the occupied channel of microcell e.mc is released ( $m c_{-} \_h[\mathbf{e} . \mathbf{m c}]$ is incremented by 1 at Step 1.15). Then the HCN tries to allocate a channel for the call, and the simulation proceeds to execute Algorithm $A$ in Fig. 17 as described in Arrival event. If the handoff call is assigned a channel (i.e., Algorithm $A$ exits from B), $N_{\mathrm{h}}$ is incremented by 1 at Step 1.16. Step 
1.17 updates the current cell for the call (i.e., e.mc $=n e w \_m c$ and e.ma $\left.=n e w \_m a\right)$ and computes the next MS move time $T_{\mathrm{h}}$ for the call. Then the simulation proceeds to execute Steps 1.9 and 1.10 (or 1.11). On the other hand, if HCN has no idle channel for the call (i.e., Algorithm $A$ exits from C), the call is forced to terminate. Then Step 1.18 is executed to increment $N_{\mathrm{f}}$ by 1 and update the $N_{\text {ff }}$ value (i.e., if this is a fast call, increment $N_{\text {ff }}$ by 1$)$.

If the call occupies a macrocell channel at Step 1.14 , then Step 1.19 checks if the MS is moving out of its macrocell e.ma. If so (i.e., new_ma $\neq \mathbf{e}$.ma), the call is handed off to the new cell. Step 1.20 increments ma_ch[e.ma] by 1; i.e., the occupied channel of e.ma is released. Then the simulation proceeds to execute Algorithm A. If the handoff call is assigned a channel (i.e., Algorithm $A$ exits from B), Steps 1.16, 1.17, 1.9 and 1.10 (or 1.11) are then executed. Otherwise, if $\mathrm{HCN}$ has no idle channel for the call (i.e., Algorithm $A$ exits from C), Step 1.18 is then executed. If the MS does not move out of its macrocell (i.e., e.ma $=n e w \_m a$ ) at Step 1.19, the simulation proceeds to execute Step 1.17 and then Steps 1.9 and 1.10 (or 1.11).

Case III. e.type $=$ Complete: At Step 1.21, if the call occupies a macrocell channel (i.e., e.is_macro $=1)$, then Step 1.22 is executed to increment $m a \_c h[$ e.ma] by 1 . Otherwise, Step 1.23 is executed to increment $m c_{-} c h[\mathbf{e} . \mathbf{m c}]$ by 1 .

To accommodate RoD-L, we only need to modify Step 2.7 of the flowchart in Fig. 17. In our simulation experiments, the confidence intervals of the $99 \%$ confidence levels are within $3 \%$ of the mean values in most cases. The simulation models are partially validated by the analytic model in [7] for the no mobility case. The details are omitted.

\section{References}

[1] R. Beraldi S. Marano C. Mastroianni, A reversible heirarchial scheme for microcellular systems with overlaying macrocells, in: Proc. IEEE Infocom, 1996, pp. 51-58.

[2] I. Chlamtac, Y. Fang, H. Zeng, Call blocking analysis for PCS networks under general cell residence time, in: IEEE
Wireless Communications and Networking Conference (WCNC), New Orleans, September 1999.

[3] Y. Fang, I. Chlamtac, Teletraffic analysis and mobility modeling for PCS network, IEEE Trans. Comm. 47 (7) (1999) 1062-1072.

[4] Y. Fang, I. Chlamtac, H.-B. Fei, Analytical results for optimal choice of location update interval for mobility database failure restoration in PCS networks, IEEE Trans. Parallel Distrib. Syst. 11 (6) (2000) 615-624.

[5] B.O.P. Gudmundson, H. Eriksson, O.E. Grumlund, Method of Effecting Handover in a Mobile Multilayer Cellular Radio System, U.S. Patent 1995.

[6] H. Holma, A. Toskala, WCDMA for UMTS, Wiley, New York, 2000.

[7] H.N. Hung, Y.-B. Lin, N.-F. Peng, H.-M. Tsai, Repacking on demand in two-tier WLL, IEEE Trans. Wireless Commun. 3 (3) (2004) 745-757.

[8] B. Jabbari, W.F. Fuhrmann, Teletraffic modeling and analysis of flexible hierarchical cellular networks with speed-sensitive handover strategy, IEEE J. Select. Areas Commun. 15 (8) (1997) 1539-1548.

[9] F.P. Kelly, Reversibility and Stochastic Networks, Wiley, New York, 1979.

[10] L. Kleinrock, Queueing Systems; Volume I: Theory, Wiley, New York, 1975.

[11] X. Lagrange, Multitier cell design, IEEE Commun. Mag. 35 (8) (1997) 60-64.

[12] Y.-B. Lin, V.W. Mak, Eliminating the boundary effect of a large-scale personal communication service network simulation, ACM Trans. Model. Comput. Simul. 4 (2) (1994) 165-190.

[13] Y.-B. Lin, W.-R. Lai, R.J. Chen, Performance analysis for dual band PCS networks, IEEE Trans. Comput. 49 (2) (2000) 148-159.

[14] K. Maheshwari, A. Kumar, Performance analysis of microcellization for supporting two mobility classes in cellular wireless networks, IEEE Trans. Vehicular Tech. 49 (2) (2000) 321-333.

[15] P.A. Ramsdale, P.S. Gaskell, Handover Techniques, U.S. Patent, 1994.

[16] S.S. Rappaport, L.-R. Hu, Microcellular communication systems with hierarchical macrocell overlays: traffic performance models and analysis, Proc. IEEE 82 (9) (1994) 1383-1397.

[17] R. Steele, M. Nofal, S. Eldolil, Adaptive algorithm for variable teletraffic demand in highway microcells, Electron. Lett. 26 (14) (1990) 988-990.

[18] H.-M. Tsai, A.-C. Pang, Y.-C. Lin, Y.-B. Lin, Repacking on demand for hierarchical cellular networks, ACM Wireless Networks, in press.

[19] F.Valois, V. Veque, Preemption policy for hierarchical cellular network, in: 5th IEEE Workshop on Mobile Multimedia Communication, 1998, pp. 75-81.

[20] F. Valois, V. Veque, QoS-oriented channel assignment strategy for hierarchical cellular networks, in: IEEE PIMRC, vol. 2, 2000, pp. 1599-1603 . 


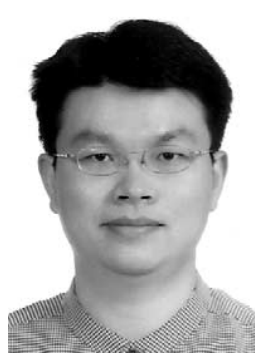

Hsien-Ming Tsai was born in Tainan, Taiwan, R.O.C., in 1973. He received the double B.S. degrees in Computer Science and Information Engineering (CSIE) and Communication Engineering, the M.S. degree in CSIE, and the Ph.D. degree in CSIE from National Chiao-Tung University (NCTU), Taiwan, in 1996, 1997, and 2002, respectively. He is currently a senior research specialist in Quanta Research Institute, Quanta Computer Inc. His research interests are in the areas of cellular protocols (UMTS/GPRS/GSM/DECT), cellular multimedia (MPEG-4 Audio/Speech), and embedded systems. He is an IEEE member.

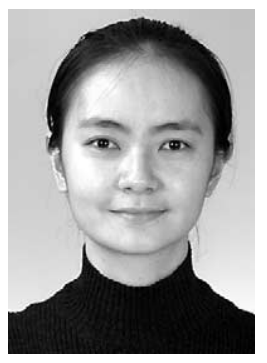

Ai-Chun Pang was born in Hsinchu, Taiwan, R.O.C., in 1973. She received the B.S., M.S. and Ph.D. degrees in Computer Science and Information Engineering from National Chiao Tung University in 1996, 1998 and 2002, respectively. She joined the Department of Computer Science and Information Engineering, National Taiwan University, Taipei, Taiwan, as an Assistant Professor in 2002. From August 2004, She also serves as an Assistant Professor in Graduate Institute of Networking and Multimedia, National Taiwan University, Taipei, Taiwan. Her research interests include design and analysis of personal communications services network, mobile computing, voice over IP and performance modeling.

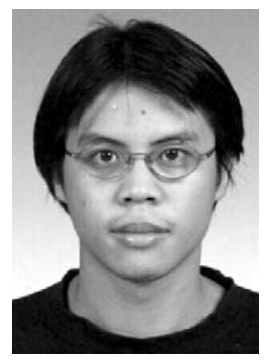

Yung-Chun Lin was born in Kaohsiung, Taiwan, R.O.C., in 1978. He received the B.S. and M.S. degrees in Computer Science and Information Engineering (CSIE) from National Chiao-Tung University (NCTU), Taiwan, in 2001, 2003, respectively. He is currently pursuing the Ph.D. degree in CSIE. His research interests include design and analysis of a personal communications services network, the cellular protocols (UMTS/GPRS/ GSM), and mobile computing.

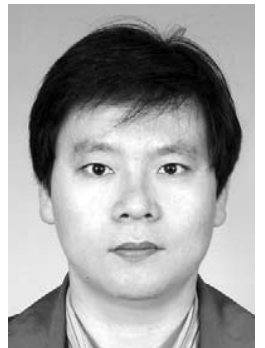

Yi-Bing Lin received his BSEE degree from National Cheng Kung University in 1983, and his Ph.D. degree in Computer Science from the University of Washington in 1990. From 1990 to 1995, he was with the Applied Research Area at Bell Communications Research (Bellcore), Morristown, NJ. In 1995, he was appointed as a professor of Department of Computer Science and Information Engineering (CSIE), National Chiao Tung University (NCTU). In 1996, he was appointed as Deputy Director of Microelectronics and Information Systems Research Center, NCTU. During 1997-1999, he was elected as Chairman of CSIE, NCTU. His current research interests include design and analysis of personal communications services network, mobile computing, distributed simulation, and performance modeling. He has published over 150 journal articles and more than 200 conference papers. $\mathrm{He}$ is an Adjunct Research Fellow of Academia Sinica, and is Chair Professor of NCTU and Providence University. He serves as consultant of many telecommunications companies including FarEasTone and Chung Hwa Telecom. He is an IEEE Fellow and an ACM Fellow. 